\title{
New Delay-Dependent Stability Criteria for Uncertain Neutral Systems with Mixed Time-Varying Delays and Nonlinear Perturbations
}

\author{
Hamid Reza Karimi, Mauricio Zapateiro, and Ningsu Luo \\ Institute of Informatics and Applications, University of Girona, Campus de Montilivi, Edifici P4, \\ 17071 Girona, Spain \\ Correspondence should be addressed to Hamid Reza Karimi, hamidreza.karimi@udg.edu
}

Received 19 July 2008; Revised 8 November 2008; Accepted 1 January 2009

Recommended by Shijun Liao

The problem of stability analysis for a class of neutral systems with mixed time-varying neutral, discrete and distributed delays and nonlinear parameter perturbations is addressed. By introducing a novel Lyapunov-Krasovskii functional and combining the descriptor model transformation, the Leibniz-Newton formula, some free-weighting matrices, and a suitable change of variables, new sufficient conditions are established for the stability of the considered system, which are neutral-delay-dependent, discrete-delay-range-dependent, and distributeddelay-dependent. The conditions are presented in terms of linear matrix inequalities (LMIs) and can be efficiently solved using convex programming techniques. Two numerical examples are given to illustrate the efficiency of the proposed method.

Copyright (C) 2009 Hamid Reza Karimi et al. This is an open access article distributed under the Creative Commons Attribution License, which permits unrestricted use, distribution, and reproduction in any medium, provided the original work is properly cited.

\section{Introduction}

Delay (or memory) systems represent a class of infinite-dimensional systems largely used to describe propagation and transport phenomena or population dynamics [1-3]. Delay differential systems are assuming an increasingly important role in many disciplines like economic, mathematics, science, and engineering. For instance, in economic systems, delays appear in a natural way since decisions and effects are separated by some time interval. The presence of a delay in a system may be the result of some essential simplification of the corresponding process model. The problem of delay effects on the stability of systems including delays in the state, and/or input is a problem of recurring interest since the delay presence may induce complex behaviors (oscillation, instability, bad performances) for the schemes $[1,2]$. Some improved methods pertaining to the problems of determining robust stability criteria and robust control design of uncertain time-delay systems have been reported; see, for example, $[4,5]$ and the references cited therein. When dealing with 
time-varying delays and the reduction of the level of design conservatism, one has to select appropriate Lyapunov-Krasovskii functional (LKF) with moderate number of terms [6].

Neutral delay systems constitute a more general class than those of the retarded type. Stability of these systems proves to be a more complex issue because the system involves the derivative of the delayed state. Especially in the past few decades, increased attention has been devoted to the problem of robust delay-independent stability or delay-dependent stability and stabilization via different approaches (e.g., model transformation techniques $[2,7-9]$, the improved bounding techniques $[10,11]$, and the properly chosen LyapunovKrasovskii functionals $[12,13])$ for a number of different neutral systems with delayed state and/or input, parameter uncertainties, and nonlinear perturbations (see, e.g., [14-25] and the references therein).

Among the existing results on neutral delay systems, the linear matrix inequality (LMI) approach is an efficient method to solve many problems such as stability analysis, stabilization $[9,15,26,27], H_{\infty}$ control problems [28-30], filter designs [31, 32], and guaranteed-cost (observer-based) control [33-39]. Besides, for neutral systems with mixed neutral and discrete delays, most of the aforementioned methods can only provide neutraldelay-independent and discrete-delay-dependent results. Furthermore, the subject of the robust stability and feedback stabilization of continuous- and discrete-time systems (within the framework LMI) under additive perturbations which are nonlinear functions in time and state of the systems are investigated in $[40,41]$, respectively.

In the recent literature on neutral systems, He et al. in [42] proposed a new approach to analyze the stability of the systems with mixed delays by incorporating some free-weighting matrices, and the less conservative criteria, which were both discrete-delay-dependent and neutral-delay-dependent, were obtained without considering the model transformations. However, some of the free matrices did not serve to reduce the conservatism of the results that were obtained. Moreover, in $[9,20]$, the authors studied the problem of the robust stability of neutral systems with nonlinear parameter perturbations and mixed time-varying neutral and discrete delays and presented neutral-delay-independent stability criteria, that cannot be directly applied to the systems with different time-varying neutral, discrete, and distributed delays. Furthermore, from the published results, it appears that general results pertaining to neutral systems with mixed time-varying neutral, discrete, and distributed delays and nonlinear parameter perturbations are few and restricted; see $[9,10,18,20,42]$ where most of the efforts were virtually neutral-delay-range-independent or were not centered on distributed delays.

In this paper, we develop new stability criteria for the stability analysis of the neutral systems with nonlinear parameter perturbations based on a descriptor model transformation. The dynamical system under consideration consists of time-varying neutral, discrete, and distributed delays without any restriction on upper bounds of derivatives of time-varying delays. By introducing a novel Lyapunov-Krasovskii functional and combining the descriptor model transformation, the Leibniz-Newton formula, some free-weighting matrices, and a suitable change of variables, new sufficient conditions are established for the stability of the considered system, which are neutral-delay-dependent, discrete-delay-range-dependent, and distributed-delay-dependent. The conditions are presented in terms of LMIs and can be easily solved by existing convex optimization techniques. Two numerical examples are given to demonstrate the less conservatism of the proposed results over some existence results in the literature. 
Notations. The superscript ' $T$ ' stands for matrix transposition; $\mathfrak{R}^{n}$ denotes the $n$ dimensional Euclidean space; $\mathfrak{R}^{n \times m}$ is the set of all real $m$ by $n$ matrices. $\|\cdot\|$ refers to the Euclidean vector norm or the induced matrix 2-norm. col $\{\cdots\}$ and diag $\{\cdots\}$ represent, respectively, a column vector and a block diagonal matrix, and the operator $\operatorname{sym}(A)$ represents $A+A^{T} \cdot \lambda_{\min }(A)$ and $\lambda_{\max }(A)$ denote, respectively, the smallest and largest eigenvalue of the square matrix $A$. The notation $P>0$ means that $P$ is real symmetric and positive definite; the symbol $*$ denotes the elements below the main diagonal of a symmetric block matrix.

\section{Problem Description}

Consider a class of linear neutral systems with different time-varying neutral, discrete, and distributed delays and nonlinear parameter perturbations represented by

$$
\begin{gathered}
\dot{x}(t)-C \dot{x}(t-\tau(t))=A x(t)+B x(t-h(t))+G_{1} f_{1}(t, x(t))+G_{2} f_{2}(t, x(t-h(t))) \\
+G_{3} \int_{t-r(t)}^{t} f_{3}(\theta, x(\theta)) d \theta+G_{4} f_{4}(t, \dot{x}(t-\tau(t))) \\
x(t)=\phi(t), \quad t \in[-\kappa, 0]
\end{gathered}
$$

where $\mathcal{\kappa}:=\max \left\{h_{2}, \tau_{1}, r_{1}\right\}$, and $x(t) \in \mathfrak{R}^{n}$ is the state vector. The time-varying vector valued initial function $\phi(t)$ is a continuously differentiable functional, and the time-varying delays $h(t), \tau(t)$, and $r(t)$ are functions satisfying, respectively,

$$
\begin{array}{cl}
0<h_{1} \leq h(t) \leq h_{2}, & |\dot{h}(t)| \leq h_{3}<\infty, \\
0<\tau(t) \leq \tau_{1}, & |\dot{\tau}(t)| \leq \tau_{2}<\infty, \\
0<r(t) \leq r_{1}, & |\dot{r}(t)| \leq r_{2}<\infty .
\end{array}
$$

The time-varying vector-valued functions $f_{i}: \mathfrak{R}^{+} \times \mathfrak{R}^{n} \rightarrow \mathfrak{R}^{n_{i}}(i=1, \ldots, 4)$ are continuous and satisfy $f_{i}(t, 0)=0$, and the Lipschitz conditions, that is, $\left\|f_{i}\left(t, x_{0}\right)-f_{i}\left(t, y_{0}\right)\right\| \leq\left\|U_{i}\left(x_{0}-y_{0}\right)\right\|$ for all $t$ and for all $x_{0}, y_{0} \in \mathfrak{R}^{n}$ such that $U_{i}$ are some known matrices.

Remark 2.1. In this case, $h(t)$ is called an interval-like or range-like time-varying delay [14]. It is also noted that this kind of time-delay describes the real situation in many practical engineering systems. For example, in the field of networked control systems, the network transmission induced delays (either from the sensor to the controller or from the controller to the plant) can be assumed to satisfy (2.2a) without loss of generality $[43,44]$.

Throughout the paper, the following assumptions are needed to enable the application of Lyapunov's method for the stability of neutral systems [1]:

(A1) let the difference operator $D: C\left([-\mathcal{\kappa}, 0], \mathfrak{R}^{n}\right) \rightarrow \mathfrak{R}^{n}$ given by $D x_{t}=x(t)-C x(t-$ $\tau(t))$ be delay-independently stable with respect to all delays. A sufficient condition for (A1) is that

(A2) all the eigenvalues of the matrix $C$ are inside the unit circle. 
Before ending this section, we recall the following lemmas, which will be used in the proof of our main results.

Lemma 2.2 (see [9]). For any arbitrary column vectors $a(s), b(s) \in \mathfrak{R}^{p}$, any matrix $W \in \mathfrak{R}^{p \times p}$, and positive-definite matrix $H \in \mathfrak{R}^{p \times p}$, the following inequality holds:

$$
-2 \int_{t-r(t)}^{t} b(s)^{T} a(s) d s \leq \int_{t-r(t)}^{t}\left[\begin{array}{l}
a(s) \\
b(s)
\end{array}\right]^{T}\left[\begin{array}{cc}
H & H W \\
* & (H W+I)^{T} H^{-1}(H W+I)
\end{array}\right]\left[\begin{array}{l}
a(s) \\
b(s)
\end{array}\right] d s .
$$

Lemma 2.3 (see [45]). Given matrices $Y=Y^{T}, D, E$, and $F$ of appropriate dimensions with $F^{T} F \leq$ $I$, then the following matrix inequality holds:

$$
Y+\operatorname{sym}(D F E)<0
$$

for all $F$ if and only if there exists a scalar $\varepsilon>0$ such that

$$
Y+\varepsilon D D^{T}+\varepsilon^{-1} E^{T} E<0 .
$$

\section{Main Results}

In this section, new delay-range-dependent sufficient conditions for the asymptotic stability of the neutral system (2.1) are presented. By utilizing the Leibniz-Newton formula, the following two zero equations hold:

$$
\begin{aligned}
& L_{1} x(t)-L_{1} x(t-h(t))-L_{1} \int_{t-h(t)}^{t} \dot{x}(s) d s=0, \\
& L_{2} x(t)-L_{2} x(t-\tau(t))-L_{2} \int_{t-\tau(t)}^{t} \dot{x}(s) d s=0,
\end{aligned}
$$

then, we can represent the system (2.1) as

$$
\begin{aligned}
\dot{x}(t)-C \dot{x}(t-\tau(t))= & \tilde{A} x(t)+\widetilde{B} x(t-h(t))+G_{1} f_{1}(t, x(t))+G_{2} f_{2}(t, x(t-h(t))) \\
& -L_{2} x(t-\tau(t))-L_{1} \int_{t-h(t)}^{t} \dot{x}(s) d s \\
& -L_{2} \int_{t-\tau(t)}^{t} \dot{x}(s) d s+G_{3} \int_{t-r(t)}^{t} f_{3}(\theta, x(\theta)) d \theta+G_{4} f_{4}(t, \dot{x}(t-\tau(t))),
\end{aligned}
$$

with $\widetilde{A}:=A+L_{1}+L_{2}$ and $\widetilde{B}:=B-L_{1}$ where the matrices $L_{1}, L_{2} \in \mathfrak{R}^{n \times n}$ will be chosen in the following theorem. 
Theorem 3.1. Under (A1), for given scalars $\gamma, h_{1}, h_{2}, \tau_{1}, r_{1}>0, h_{3}, \tau_{2}, r_{2}$, the neutral system (2.1) is asymptotically stable, if there exist some scalars $\delta, \alpha_{1}, \alpha_{2}$, matrices $P_{2},\left\{N_{i}\right\}_{i=1}^{20}, Y_{1}, Y_{2}$, and positivedefinite matrices $P_{1},\left\{Q_{i}\right\}_{i=1}^{4},\left\{R_{i}\right\}_{i=1}^{4}, \widetilde{H}_{1}, \widetilde{H}_{2}$, such that the following $L M I$ is feasible:

$\Pi=\left[\begin{array}{ccccccccccc}\Pi_{11} & \Pi_{12} & \Pi_{13} & \Pi_{14} & \Pi_{15} & \Pi_{16} & \Pi_{17} & \Pi_{18} & \Pi_{19} & \Pi_{1,10} & \Pi_{1,11} \\ * & \Pi_{22} & \Pi_{23} & 0 & -B^{T} N_{17}^{T} & 0 & \Pi_{27} & -B^{T} N_{19}^{T} & \Pi_{29} & \Pi_{2,10} & 0 \\ * & * & \Pi_{33} & 0 & 0 & 0 & \Pi_{37} & 0 & \Pi_{39} & 0 & 0 \\ * & * & * & \Pi_{44} & 0 & 0 & -N_{15}^{T} & 0 & -N_{16}^{T} & \Pi_{4,10} & 0 \\ * & * & * & * & \Pi_{55} & \Pi_{56} & -C^{T} N_{18}^{T} & -N_{17} G_{3} & -C^{T} N_{20}^{T} & 0 & 0 \\ * & * & * & * & * & \Pi_{66} & \Pi_{67} & \Pi_{68} & \Pi_{69} & 0 & 0 \\ * & * & * & * & * & * & \Pi_{77} & -N_{18} G_{3} & -N_{18} G_{4} & \Pi_{7,10} & 0 \\ * & * & * & * & * & * & * & \Pi_{88} & -G_{3}^{T} N_{20}^{T} & 0 & 0 \\ * & * & * & * & * & * & * & * & -\operatorname{sym}\left(N_{20} G_{4}\right) & \Pi_{9,10} & 0 \\ * & * & * & * & * & * & * & * & * & \Pi_{10,10} & 0 \\ * & * & * & * & * & * & * & * & * & * & \Pi_{11,11}\end{array}\right]<0$

with

$$
\begin{aligned}
& \Pi_{11}=\operatorname{sym}\left(\left[\begin{array}{cc}
P_{2}^{T} A+\left(1+\alpha_{1}\right) Y_{1}+\left(1+\alpha_{2}\right) Y_{2} & P_{1}-P_{2}^{T} \\
\delta\left(P_{2}^{T} A+\left(1+\alpha_{1}\right) Y_{1}+\left(1+\alpha_{2}\right) Y_{2}\right) & -\delta P_{2}^{T}
\end{array}\right]\right)+\Omega, \\
& \Pi_{12}=\left[\begin{array}{c}
P_{2}^{T} B-\left(1+\alpha_{1}\right) Y_{1}+N_{2}^{T}-N_{1}-N_{5}+N_{9} \\
\delta P_{2}^{T} B-\delta\left(1+\alpha_{1}\right) Y_{1}
\end{array}\right], \quad \Pi_{13}=\left[\begin{array}{cc}
N_{5} & N_{9} \\
0 & 0
\end{array}\right], \\
& \Pi_{14}=\left[\begin{array}{c}
-\left(1+\alpha_{2}\right) Y_{2}+N_{14}^{T}-N_{13} \\
-\delta\left(1+\alpha_{2}\right) Y_{2}
\end{array}\right], \quad \Pi_{15}=\left[\begin{array}{c}
P_{2}^{T} C-A^{T} N_{17}^{T} \\
\delta P_{2}^{T} C+N_{17}^{T}
\end{array}\right], \\
& \Pi_{16}=\left[\begin{array}{cc}
P_{2}^{T} G_{1} & P_{2}^{T} G_{2} \\
\delta P_{2}^{T} G_{1} & \delta P_{2}^{T} G_{2}
\end{array}\right], \quad \Pi_{17}=\left[\begin{array}{c}
N_{3}^{T}+N_{15}^{T}-A^{T} N_{18}^{T} \\
N_{18}^{T}
\end{array}\right], \\
& \Pi_{18}=\left[\begin{array}{c}
P_{2}^{T} G_{3}-A^{T} N_{19}^{T} \\
\delta P_{2}^{T} G_{3}+N_{19}^{T}
\end{array}\right], \quad \Pi_{19}=\left[\begin{array}{c}
P_{2}^{T} G_{4}+N_{4}^{T}+N_{16}^{T}-A^{T} N_{20}^{T} \\
\delta P_{2}^{T} G_{4}+N_{20}^{T}
\end{array}\right], \\
& \Pi_{1,10}=\left[\begin{array}{cccc}
h_{12} N_{1} & h_{12} N_{5} & h_{12} N_{9} & \tau_{1} N_{13} \\
0 & 0 & 0 & 0
\end{array}\right],
\end{aligned}
$$




$$
\begin{aligned}
& \left.\Pi_{1,11}=\left[\begin{array}{lll}
h_{2}\left(\alpha_{1}+1\right) \widetilde{H}_{1} & \tau_{1}\left(\alpha_{1}+1\right) \widetilde{H}_{2} & h_{2} \\
I
\end{array}\right]\left[\begin{array}{c}
Y_{1} \\
\delta Y_{1}
\end{array}\right]^{T} \tau_{1}\left[\begin{array}{l}
0 \\
I
\end{array}\right]\left[\begin{array}{c}
Y_{2} \\
\delta Y_{2}
\end{array}\right]^{T}\right], \\
& \Pi_{22}=U_{2}^{T} U_{2}-\left(1-h_{3}\right) R_{3}-\operatorname{sym}\left(N_{2}+N_{6}-N_{10}\right), \quad \Pi_{23}=\left[\begin{array}{ll}
N_{6} & N_{10}
\end{array}\right], \\
& \Pi_{27}=-N_{3}^{T}-N_{7}^{T}+N_{11}^{T}-B^{T} N_{18}^{T}, \quad \Pi_{29}=-N_{4}^{T}-N_{8}^{T}+N_{12}^{T}-B^{T} N_{20}^{T},
\end{aligned}
$$$$
\Pi_{2,10}=\left[\begin{array}{llll}
h_{12} N_{2} & h_{12} N_{6} & h_{12} N_{10} & 0
\end{array}\right], \quad \Pi_{33}=\operatorname{diag}\left\{-R_{1},-R_{2}\right\}, \quad \Pi_{37}=\left[\begin{array}{c}
N_{7}^{T} \\
-N_{11}^{T}
\end{array}\right],
$$$$
\Pi_{39}=\left[\begin{array}{c}
N_{8}^{T} \\
-N_{12}^{T}
\end{array}\right], \quad \Pi_{44}=-\left(1-\tau_{2}\right) Q_{1}-\operatorname{sym}\left(N_{14}\right), \quad \Pi_{4,10}=\left[\begin{array}{llll}
0 & 0 & 0 & \tau_{1} N_{14}
\end{array}\right]
$$$$
\Pi_{55}=-\left(1-\tau_{2}\right) Q_{2}-\operatorname{sym}\left(N_{17} C\right)+U_{4}^{T} U_{4}, \quad \Pi_{56}=\left[\begin{array}{lll}
-N_{17} G_{1} & -N_{17} G_{2}
\end{array}\right],
$$$$
\Pi_{66}=\operatorname{diag}\{-I,-I\}, \quad \Pi_{67}=\left[\begin{array}{c}
-G_{1}^{T} N_{18}^{T} \\
-G_{2}^{T} N_{18}^{T}
\end{array}\right], \quad \Pi_{68}=\left[\begin{array}{ll}
N_{19} G_{1} & N_{19} G_{2}
\end{array}\right]^{T},
$$$$
\Pi_{69}=\left[\begin{array}{c}
-G_{1}^{T} N_{20}^{T} \\
-G_{2}^{T} N_{20}^{T}
\end{array}\right], \quad \Pi_{77}=-I+r_{1}^{2} Q_{4}, \quad \Pi_{88}=-\left(1-r_{2}\right) Q_{4}-\operatorname{sym}\left(N_{19} G_{3}\right),
$$

$$
\begin{aligned}
& \Pi_{4,10}=\left[\begin{array}{llll}
0 & 0 & 0 & \tau_{1} N_{14}
\end{array}\right], \quad \Pi_{7,10}=\left[\begin{array}{lllll}
h_{12} N_{3} & h_{12} N_{7} & h_{12} N_{11} & \tau_{1} N_{15}
\end{array}\right], \\
& \Pi_{9,10}=\left[\begin{array}{llll}
h_{12} N_{4} & h_{12} N_{8} & h_{12} N_{12} & \tau_{1} N_{16}
\end{array}\right], \\
& \Pi_{10,10}=\operatorname{diag}\left\{-h_{12} R_{4},-h_{12} R_{5},-h_{12} R_{4},-\tau_{1} Q_{3}\right\}, \\
& \Pi_{1,11}=\operatorname{diag}\left\{-h_{2} \widetilde{H}_{1},-\tau_{1} \widetilde{H}_{2},-h_{2} \widetilde{H}_{1},-\tau_{1} \widetilde{H}_{2}\right\},
\end{aligned}
$$

where $\Omega=\operatorname{diag}\left\{Q_{1}+\sum_{i=1}^{3} R_{i}+U_{1}^{T} U_{1}+U_{3}^{T} U_{3}+\operatorname{sym}\left(N_{1}+N_{13}\right), Q_{2}+\tau_{1} Q_{3}+h_{12} R_{4}+h_{2} R_{5}\right\}$. Proof. Firstly, we represent (3.2) in an equivalent descriptor model form as

$$
\begin{gathered}
\dot{x}(t)=\eta(t), \\
0=-\eta(t)+\widetilde{A} x(t)+C \eta(t-\tau(t))+\widetilde{B} x(t-h(t))-L_{2} x(t-\tau(t)) \\
+G_{1} f_{1}(t, x(t))+G_{2} f_{2}(t, x(t-h(t)))-L_{1} \int_{t-h(t)}^{t} \eta(s) d s-L_{2} \int_{t-\tau(t)}^{t} \eta(s) d s \\
+G_{3} \int_{t-r(t)}^{t} f_{3}(\theta, x(\theta)) d \theta+G_{4} f_{4}(t, \eta(t-\tau(t))) .
\end{gathered}
$$


Define the Lyapunov-Krasovskii functional

$$
V(t)=\sum_{i=1}^{6} V_{i}(t)
$$

where

$$
\begin{aligned}
V_{1}(t)= & x(t)^{T} P_{1} x(t):=\xi(t)^{T} T P \xi(t), \\
V_{2}(t)= & \int_{t-\tau(t)}^{t}\left[x(s)^{T} Q_{1} x(s)+\eta(s)^{T} Q_{2} \eta(s)\right] d s \\
& +\int_{t-h(t)}^{t} x(s)^{T} R_{3} x(s) d s+\sum_{i=1}^{2} \int_{t-h_{i}}^{t} x(s)^{T} R_{i} x(s) d s, \\
V_{3}(t)= & \int_{-h_{2}}^{-h_{1}} \int_{t+\theta}^{t} \eta(s)^{T} R_{4} \eta(s) d s d \theta+\int_{-h_{2}}^{0} \int_{t+\theta}^{t} \eta(s)^{T} R_{5} \eta(s) d s d \theta, \\
V_{4}(t)= & \int_{-\tau_{1}}^{0} \int_{t+\theta}^{t} \eta(s)^{T} Q_{3} \eta(s) d s d \theta, \\
V_{5}(t)= & \left.\int_{-h_{2}}^{0} \int_{t+\theta}^{t} \eta(s)^{T}\left[\begin{array}{c}
0 \\
L_{1}
\end{array}\right]^{T} H_{1}[0] L_{1}\right] \eta(s) d s d \theta \\
& \left.+\int_{-\tau_{1}}^{0} \int_{t+\theta}^{t} \eta(s)^{T}[0]^{T} H_{2}[0] L_{2}\right]^{t} \eta(s) d s d \theta, \\
V_{6}(t)= & \int_{t-r(t)}^{t}\left[\int_{s}^{t} f_{3}(\theta, x(\theta))^{T} d \theta\right] Q_{4}\left[\int_{s}^{t} f_{3}(\theta, x(\theta))^{T} d \theta\right] d s \\
& +\int_{0}^{r_{1}} \int_{t-s}^{t}(\theta-t+s) f_{3}(\theta, x(\theta))^{T} Q_{4} f_{3}(\theta, x(\theta)) d \theta d s,
\end{aligned}
$$

with $\xi(t):=\operatorname{col}\{x(t), \eta(t)\}, T=\operatorname{diag}\{I, 0\}$, and $P=\left[\begin{array}{ll}P_{1} & 0 \\ P_{2} & P_{3}\end{array}\right]$, where $P_{1}=P_{1}^{T}>0$.

On the other hand, noting that $V(\phi(t), t) \geq \lambda_{\min }\left(P_{1}\right)\|\phi(0)\|^{2}$. According to [34], using the Cauchy-Schwarz inequality and after some manipulations, we obtain

$$
V(\phi(t), t) \leq V(\phi(0), 0) \leq \rho\left[\|\phi(0)\|^{2}+\int_{-\kappa}^{0}\|\dot{\phi}(\theta)\|^{2} d \theta\right],
$$

where $\rho:=\max \left(\rho_{1}, \rho_{2}\right)$ with

$$
\begin{aligned}
\rho_{1}:= & \lambda_{\max }\left(P_{1}\right)+2 \tau_{1} \lambda_{\max }\left(Q_{1}\right)+2 h_{1} \lambda_{\max }\left(R_{1}\right) \\
& +2 h_{2} \lambda_{\max }\left(R_{2}\right)+2 h_{2} \lambda_{\max }\left(R_{3}\right)+3 r_{1}^{2} \lambda_{\max }\left(U_{3}^{T} Q_{4} U_{3}\right),
\end{aligned}
$$




$$
\begin{aligned}
\rho_{2}:= & 2 \tau_{1}^{2} \lambda_{\max }\left(Q_{1}\right)+\lambda_{\max }\left(Q_{2}\right)+2 h_{2}^{2} \lambda_{\max }\left(R_{3}\right)+2 h_{1}^{2} \lambda_{\max }\left(R_{1}\right)+2 h_{2}^{2} \lambda_{\max }\left(R_{2}\right) \\
& +h_{2} \lambda_{\max }\left(R_{5}\right)+\left(h_{1}+h_{2}\right) \lambda_{\max }\left(R_{4}\right)+\tau_{1} \lambda_{\max }\left(Q_{3}\right) \\
& +h_{2} \lambda_{\max }\left(\left[\begin{array}{c}
0 \\
L_{1}
\end{array}\right]^{T} H_{1}\left[\begin{array}{c}
0 \\
L_{1}
\end{array}\right]\right)+\tau_{1} \lambda_{\max }\left(\left[\begin{array}{c}
0 \\
L_{2}
\end{array}\right]^{T} H_{2}\left[\begin{array}{c}
0 \\
L_{2}
\end{array}\right]\right)+\frac{11}{3} r_{1}^{3} \lambda_{\max }\left(U_{3}^{T} Q_{4} U_{3}\right) .
\end{aligned}
$$

Differentiating $V_{1}(t)$ along the system trajectory becomes

$$
\begin{aligned}
& \dot{V}_{1}(t)=2 x(t)^{T} P_{1} \dot{\xi}(t) \\
& =2 \xi(t)^{T} P^{T}\left[\begin{array}{c}
\dot{x}(t) \\
0
\end{array}\right] \\
& =2 \xi(t)^{T} P^{T}\left\{\bar{A} \xi(t)+\left[\begin{array}{l}
0 \\
C
\end{array}\right] \eta(t-\tau(t))+\left[\begin{array}{c}
0 \\
\widetilde{B}
\end{array}\right] x(t-h(t))-\left[\begin{array}{c}
0 \\
L_{2}
\end{array}\right] x(t-\tau(t))\right. \\
& +\left[\begin{array}{c}
0 \\
G_{1}
\end{array}\right] f_{1}(t, x(t))+\left[\begin{array}{c}
0 \\
G_{2}
\end{array}\right] f_{2}(t, x(t-h(t))) \\
& \left.+G_{3} \int_{t-r(t)}^{t} f_{3}(\theta, x(\theta)) d \theta+G_{4} f_{4}(t, \eta(t-\tau(t)))\right\}+\beta_{1}(t)+\beta_{2}(t),
\end{aligned}
$$

where

$$
\begin{gathered}
\bar{A}:=\left[\begin{array}{cc}
0 & I \\
\tilde{A} & -I
\end{array}\right], \quad \beta_{1}(t)=-2 \int_{t-h(t)}^{t} \xi(t)^{T} P^{T}\left[\begin{array}{c}
0 \\
L_{1}
\end{array}\right] \eta(s) d s, \\
\beta_{2}(t)=-2 \int_{t-\tau(t)}^{t} \xi(t)^{T} P^{T}\left[\begin{array}{c}
0 \\
L_{2}
\end{array}\right] \eta(s) d s .
\end{gathered}
$$

Using Lemma 2.2 for $a(s)=\operatorname{col}\left\{0, L_{i}\right\} \xi(s)$ and $b=P \operatorname{col}\{\xi(t), \eta(t)\}$, we obtain

$$
\begin{aligned}
\beta_{1}(t) \leq & h_{2} \xi(t)^{T} P^{T}\left(W_{1}^{T} H_{1}+I\right)^{T} H_{1}^{-1}\left(W_{1}^{T} H_{1}+I\right) P \xi(t) \\
& +2 \xi(t)^{T} P^{T} W_{1}^{T} H_{1}\left[\begin{array}{c}
0 \\
L_{1}
\end{array}\right](x(t)-x(t-h(t))) \\
& +\int_{t-h_{2}}^{t} \eta(s)^{T}\left[\begin{array}{c}
0 \\
L_{1}
\end{array}\right]^{T} H_{1}\left[\begin{array}{c}
0 \\
L_{1}
\end{array}\right] \eta(s) d s, \\
\beta_{2}(t) \leq & \tau_{1} \xi(t)^{T} P^{T}\left(W_{2}^{T} H_{2}+I\right)^{T} H_{2}^{-1}\left(W_{2}^{T} H_{2}+I\right) P \xi(t) \\
& +2 \xi(t)^{T} P^{T} W_{2}^{T} H_{2}\left[\begin{array}{c}
0 \\
L_{2}
\end{array}\right](x(t)-x(t-\tau(t))) \\
& +\int_{t-\tau_{1}}^{t} \eta(s)^{T}\left[\begin{array}{c}
0 \\
L_{2}
\end{array}\right]^{T} H_{2}\left[\begin{array}{c}
0 \\
L_{2}
\end{array}\right] \eta(s) d s .
\end{aligned}
$$


Differentiating the second Lyapunov term in (3.6) gives

$$
\begin{aligned}
\dot{V}_{2}(t)= & x(t)^{T}\left(Q_{1}+\sum_{i=1}^{3} R_{i}\right) x(t)+\eta(t)^{T} Q_{2} \eta(t)-(1-\dot{h}(t)) x^{T}(t-h(t)) R_{3} x(t-h(t)) \\
& -(1-\dot{\tau}(t)) x^{T}(t-\tau(t)) Q_{1} x(t-\tau(t)) \\
& -(1-\dot{\tau}(t)) \eta^{T}(t-\tau(t)) Q_{2} \eta(t-\tau(t))-\sum_{i=1}^{2} x\left(t-h_{i}\right)^{T} R_{i} x\left(t-h_{i}\right) \\
\leq & x(t)^{T}\left(Q_{1}+\sum_{i=1}^{3} R_{i}\right) x(t)+\eta(t)^{T} Q_{2} \eta(t)-\left(1-h_{3}\right) x^{T}(t-h(t)) R_{3} x(t-h(t)) \\
& -\left(1-\tau_{2}\right) x^{T}(t-\tau(t)) Q_{1} x(t-\tau(t)) \\
& -\left(1-\tau_{2}\right) \eta^{T}(t-\tau(t)) Q_{2} \eta(t-\tau(t))-\sum_{i=1}^{2} x\left(t-h_{i}\right)^{T} R_{i} x\left(t-h_{i}\right),
\end{aligned}
$$

and the time derivative of the third term of $V(t)$ in (3.6) is

$$
\begin{aligned}
\dot{V}_{3}(t) & =\eta(t)^{T}\left(h_{12} R_{4}+h_{2} R_{5}\right) \eta(t)-\int_{t-h_{2}}^{t-h_{1}} \eta(s)^{T} R_{4} \eta(s) d s-\int_{t-h_{2}}^{t} \eta(s)^{T} R_{5} \eta(s) d s \\
& \leq \eta(t)^{T}\left(h_{12} R_{4}+h_{2} R_{5}\right) \eta(t)-\int_{t-h_{2}}^{t-h(t)} \eta(s)^{T} R_{4} \eta(s) d s-\int_{t-h(t)}^{t-h_{1}} \eta(s)^{T}\left(R_{4}+R_{5}\right) \eta(s) d s,
\end{aligned}
$$

and, similarly,

$$
\dot{V}_{4}(t)=\tau_{1} \eta(t)^{T} Q_{3} \eta(t)-\int_{t-\tau_{1}}^{t} \eta(s)^{T} Q_{3} \eta(s) d s \leq \tau_{1} \eta(t)^{T} Q_{3} \eta(t)-\int_{t-\tau(t)}^{t} \eta(s)^{T} Q_{3} \eta(s) d s,
$$

and also the time derivative of the fifth and sixth terms of $V(t)$ in (3.6) are, respectively,

$$
\begin{aligned}
\dot{V}_{5}(t)= & \eta(t)^{T}\left(h_{2}\left[\begin{array}{c}
0 \\
L_{1}
\end{array}\right]^{T} H_{1}\left[\begin{array}{c}
0 \\
L_{1}
\end{array}\right]+\tau_{1}\left[\begin{array}{c}
0 \\
L_{2}
\end{array}\right]^{T} H_{2}\left[\begin{array}{c}
0 \\
L_{2}
\end{array}\right]\right) \eta(t) \\
& -\int_{t-h_{2}}^{t} \eta(s)^{T}\left[\begin{array}{c}
0 \\
L_{1}
\end{array}\right]^{T} H_{1}\left[\begin{array}{c}
0 \\
L_{1}
\end{array}\right] \eta(s) d s-\int_{t-\tau_{1}}^{t} \eta(s)^{T}\left[\begin{array}{c}
0 \\
L_{2}
\end{array}\right]^{T} H_{2}\left[\begin{array}{c}
0 \\
L_{2}
\end{array}\right] \eta(s) d s,
\end{aligned}
$$




$$
\begin{aligned}
\dot{V}_{6}(t)= & -(1-\dot{r}(t))\left[\int_{t-r(t)}^{t} f_{3}(\theta, x(\theta))^{T} d \theta\right] Q_{4}\left[\int_{t-r(t)}^{t} f_{3}(\theta, x(\theta)) d \theta\right] \\
& +2 \int_{t-r(t)}^{t} f_{3}(t, x(t))^{T} Q_{4}\left[\int_{s}^{t} f_{3}(\theta, x(\theta)) d \theta\right] d s \\
& +\int_{0}^{r_{1}} s f_{3}(t, x(t))^{T} Q_{4} f_{3}(t, x(t)) d s-\int_{0}^{r_{1}} \int_{t-s}^{t} f_{3}(\theta, x(\theta))^{T} Q_{4} f_{3}(\theta, x(\theta)) d \theta d s \\
\leq & \int_{t-r(t)}^{t}(\theta-t+r(t))\left[f_{3}(t, x(t))^{T} Q_{4} f_{3}(t, x(t))+f_{3}(\theta, x(\theta))^{T} Q_{4} f_{3}(\theta, x(\theta))\right] d \theta \\
& +\int_{0}^{r_{1}} s f_{3}(t, x(t))^{T} Q_{4} f_{3}(t, x(t)) d s \\
& -\left(1-r_{2}\right)\left[\int_{t-r(t)}^{t} f_{3}(\theta, x(\theta))^{T} d \theta\right] Q_{4}\left[\int_{t-r(t)}^{t} f_{3}(\theta, x(\theta)) d \theta\right] \\
& -\int_{t-r_{1}}^{t}\left(\theta-t+r_{1}\right) f_{3}(\theta, x(\theta))^{T} Q_{4} f_{3}(\theta, x(\theta)) d \theta \\
= & r_{1}^{2} f_{3}(t, x(t))^{T} Q_{4} f_{3}(t, x(t))-\left(1-r_{2}\right)\left[\int_{t-r(t)}^{t} f_{3}(\theta, x(\theta))^{T} d \theta\right] Q_{4}\left[\int_{t-r(t)}^{t} f_{3}(\theta, x(\theta)) d \theta\right] .
\end{aligned}
$$

For nonlinear functions $f_{i}(\cdot)$, we have

$$
\begin{gathered}
0 \leq-f_{1}(t, x(t))^{T} f_{1}(t, x(t))+x(t)^{T} U_{1}^{T} U_{1} x(t), \\
0 \leq-f_{2}(t, x(t-h(t)))^{T} f_{2}(t, x(t-h(t)))+x(t-h(t))^{T} U_{2}^{T} U_{2} x(t-h(t)), \\
0 \leq-f_{3}(t, x(t))^{T} f_{3}(t, x(t))+x(t)^{T} U_{3}^{T} U_{3} x(t), \\
0=-f_{4}(t, \eta(t-\tau(t)))^{T} f_{4}(t, \eta(t-\tau(t)))+\eta(t-\tau(t))^{T} U_{4}^{T} U_{4} \eta(t-\tau(t)) .
\end{gathered}
$$

Moreover, from the Leibniz-Newton formula and the system (2.1), the following equations hold for any matrices $\left\{N_{i}\right\}_{i=1}^{10}$ with appropriate dimensions:

$$
\begin{aligned}
& 2 \mho^{T}(t) \chi_{1}\left(x(t)-x(t-h(t))-\int_{t-h(t)}^{t} \eta(s) d s\right)=0 \\
& 2 \vartheta^{T}(t) \chi_{2}\left(x\left(t-h_{1}\right)-x(t-h(t))-\int_{t-h(t)}^{t-h_{1}} \eta(s) d s\right)=0 \\
& 2 \vartheta^{T}(t) X_{3}\left(x(t-h(t))-x\left(t-h_{2}\right)-\int_{t-h_{2}}^{t-h(t)} \eta(s) d s\right)=0 \\
& 2 \mho^{T}(t) \chi_{4}\left(x(t)-x(t-\tau(t))-\int_{t-\tau(t)}^{t} \eta(s) d s\right)=0
\end{aligned}
$$


Mathematical Problems in Engineering

$$
\begin{gathered}
2 \mho^{T}(t) X_{5}\left(\eta(t)-C \eta(t-\tau(t))-A x(t)-B x(t-h(t))-G_{1} f_{1}(t, x(t))-G_{2} f_{2}(t, x(t-h(t)))\right. \\
\left.-G_{3} \int_{t-r(t)}^{t} f_{3}(\theta, x(\theta)) d \theta-G_{4} f_{4}(t, \eta(t-\tau(t)))\right)=0,
\end{gathered}
$$

where

$$
\begin{aligned}
& X_{1}:= {[N_{1}^{T}, 0, N_{2}^{T}, \underbrace{0, \ldots, 0}_{6 \text { elements }}, N_{3}^{T}, 0, N_{4}^{T}]^{T}, } \\
& X_{2}:= {[N_{5}^{T}, 0, N_{6}^{T}, \underbrace{0, \ldots, 0}_{6 \text { elements }}, N_{7}^{T}, 0, N_{8}^{T}]^{T} } \\
& X_{3}:= {[N_{9}^{T}, 0, N_{10}^{T}, \underbrace{0, \ldots, 0}_{6 \text { elements }}, N_{11}^{T}, 0, N_{12}^{T}]^{T}, } \\
& X_{4}:= {[N_{13}^{T}, \underbrace{0, \ldots, 0}_{4 \text { elements }}, N_{14}^{T}, \underbrace{0, \ldots, 0}_{3 \text { elements }}, N_{15}^{T}, 0, N_{16}^{T}]^{T}, } \\
& X_{5}:= {[\underbrace{0, \ldots, 0}_{6 \text { elements }}, N_{17}^{T}, 0,0, N_{18}^{T}, N_{19}^{T}, N_{20}^{T}]^{T}, } \\
& \vartheta(t):=\operatorname{col}\left\{x(t), \eta(t), x(t-h(t)), x\left(t-h_{1}\right), x\left(t-h_{2}\right), x(t-\tau(t)),\right. \\
& \eta(t-\tau(t)), f_{1}(t, x(t)), f_{2}(t, x(t-h(t))), f_{3}(t, x(t)), \\
&\left.\int_{t-r(t)}^{t} f_{3}(\theta, x(\theta)) d \theta, f_{4}(t, \eta(t-\tau(t)))\right\} .
\end{aligned}
$$

Using the obtained derivative terms (3.10)-(3.17) and adding the right- and the left-hand sides of (3.18) and (3.19) into $\dot{V}(t)$, the following result is obtained:

$$
\begin{aligned}
\dot{V}(t)= & \sum_{i=1}^{6} \dot{V}_{i}(t) \\
\leq & \vartheta(t)^{T} \Sigma \vartheta(t)-\int_{t-h(t)}^{t-h_{1}}\left(\vartheta^{T}(t) \chi_{1}+\eta^{T}(s) R_{4}\right) R_{4}^{-1}\left(\vartheta^{T}(t) \chi_{1}+\eta^{T}(s) R_{4}\right)^{T} d s \\
& -\int_{t-h(t)}^{t-h_{1}}\left(\vartheta^{T}(t) X_{2}+\eta^{T}(s) R_{5}\right) R_{5}^{-1}\left(\vartheta^{T}(t) \chi_{2}+\eta^{T}(s) R_{5}\right)^{T} d s \\
& -\int_{t-h(t)}^{t-h_{1}}\left(\vartheta^{T}(t) X_{3}+\eta^{T}(s) R_{4}\right) R_{4}^{-1}\left(\vartheta^{T}(t) \chi_{3}+\eta^{T}(s) R_{5}\right)^{T} d s \\
& -\int_{t-\tau(t)}^{t}\left(\vartheta^{T}(t) X_{4}+\eta^{T}(s) Q_{3}\right) Q_{3}^{-1}\left(\vartheta^{T}(t) \chi_{4}+\eta^{T}(s) Q_{3}\right)^{T} d s
\end{aligned}
$$


where $\Sigma:=\widehat{\Pi}+h_{12} \chi_{1} R_{4}^{-1} \chi_{1}^{T}+h_{12} \chi_{2} R_{5}^{-1} \chi_{2}^{T}+h_{12} \chi_{3} R_{4}^{-1} \chi_{3}^{T}+\tau_{1} \chi_{4} Q_{3}^{-1} \chi_{4}^{T}$, and the matrix $\widehat{\Pi}$ is given by

$$
\widehat{\Pi}=\left[\begin{array}{ccccccccc}
\widehat{\Pi}_{11} & \widehat{\Pi}_{12} & \Pi_{13} & \widehat{\Pi}_{14} & \widehat{\Pi}_{15} & \widehat{\Pi}_{16} & \Pi_{17} & \widehat{\Pi}_{18} & \widehat{\Pi}_{19} \\
* & \Pi_{22} & \Pi_{23} & 0 & -B^{T} N_{17}^{T} & 0 & \Pi_{27} & -B^{T} N_{19}^{T} & \Pi_{29} \\
* & * & \Pi_{33} & 0 & 0 & 0 & \Pi_{37} & 0 & \Pi_{39} \\
* & * & * & \Pi_{44} & 0 & 0 & -N_{15}^{T} & 0 & -N_{16}^{T} \\
* & * & * & * & \Pi_{55} & \Pi_{56} & -C^{T} N_{18}^{T} & -N_{17} G_{3} & -C^{T} N_{20}^{T} \\
* & * & * & * & * & \Pi_{66} & \Pi_{67} & \Pi_{68} & \Pi_{69} \\
* & * & * & * & * & * & \Pi_{77} & -N_{18} G_{3} & -N_{18} G_{4} \\
* & * & * & * & * & * & * & \Pi_{88} & -G_{3}^{T} N_{20}^{T} \\
* & * & * & * & * & * & * & * & -\operatorname{sym}\left(N_{20} G_{4}\right)
\end{array}\right]
$$

with

$$
\begin{aligned}
& \widehat{\Pi}_{11}=\operatorname{sym}\left(P^{T}\left(\bar{A}+\left(W_{1}^{T} H_{1}\left[\begin{array}{c}
0 \\
L_{1}
\end{array}\right]+W_{2}^{T} H_{2}\left[\begin{array}{c}
0 \\
L_{2}
\end{array}\right]\right)\left[\begin{array}{ll}
I & 0
\end{array}\right]\right)\right) \\
& +P^{T}\left[h_{2}\left(W_{1}^{T} H_{1}+I\right)^{T} H_{1}^{-1}\left(W_{1}^{T} H_{1}+I\right)\right. \\
& \left.+\tau_{1}\left(W_{2}^{T} H_{2}+I\right)^{T} H_{2}^{-1}\left(W_{2}^{T} H_{2}+I\right)\right] P \\
& +\left[\begin{array}{l}
0 \\
I
\end{array}\right]\left(h_{2}\left[\begin{array}{c}
0 \\
L_{1}
\end{array}\right]^{T} H_{1}\left[\begin{array}{c}
0 \\
L_{1}
\end{array}\right]+\tau_{1}\left[\begin{array}{c}
0 \\
L_{2}
\end{array}\right]^{T} H_{2}\left[\begin{array}{c}
0 \\
L_{2}
\end{array}\right]\right)\left[\begin{array}{l}
0 \\
I
\end{array}\right]^{T}+\Omega \\
& \widehat{\Pi}_{12}=P^{T}\left[\begin{array}{c}
0 \\
\tilde{B}
\end{array}\right]-P^{T} W_{1}^{T} H_{1}\left[\begin{array}{c}
0 \\
L_{1}
\end{array}\right]+\left[\begin{array}{c}
N_{2}^{T}-N_{1}-N_{5}+N_{9} \\
0
\end{array}\right], \\
& \widehat{\Pi}_{14}=-P^{T}\left[\begin{array}{c}
0 \\
L_{2}
\end{array}\right]-P^{T} W_{2}^{T} H_{2}\left[\begin{array}{c}
0 \\
L_{2}
\end{array}\right]+\left[\begin{array}{c}
N_{14}^{T}-N_{13} \\
0
\end{array}\right], \quad \widehat{\Pi}_{15}=P^{T}\left[\begin{array}{l}
0 \\
C
\end{array}\right]+\left[\begin{array}{c}
-A^{T} N_{17}^{T} \\
N_{17}^{T}
\end{array}\right] \text {, } \\
& \widehat{\Pi}_{16}=P^{T}\left[\begin{array}{cc}
0 & 0 \\
G_{1} & G_{2}
\end{array}\right], \quad \widehat{\Pi}_{18}=P^{T}\left[\begin{array}{c}
0 \\
G_{3}
\end{array}\right]+\left[\begin{array}{c}
-A^{T} N_{19}^{T} \\
N_{19}^{T}
\end{array}\right], \\
& \widehat{\Pi}_{19}=P^{T}\left[\begin{array}{c}
0 \\
G_{4}
\end{array}\right]+\left[\begin{array}{c}
N_{4}^{T}+N_{16}^{T}-A^{T} N_{20}^{T} \\
N_{20}^{T}
\end{array}\right] .
\end{aligned}
$$


Now, if $\Sigma<0$ holds, then $\dot{V}(t)<0$ which means that the neutral system (2.1) is asymptotically stable. By applying the Schur complement, the matrix inequity $\Sigma<0$ results in

$$
\left[\begin{array}{ccccccccccc}
\tilde{\Pi}_{11} & \widehat{\Pi}_{12} & \Pi_{13} & \widehat{\Pi}_{14} & \widehat{\Pi}_{15} & \widehat{\Pi}_{16} & \Pi_{17} & \widehat{\Pi}_{18} & \widehat{\Pi}_{19} & \Pi_{1,10} & \widehat{\Pi}_{1,11} \\
* & \Pi_{22} & \Pi_{23} & 0 & -B^{T} N_{17}^{T} & 0 & \Pi_{27} & -B^{T} N_{19}^{T} & \Pi_{29} & \Pi_{2,10} & 0 \\
* & * & \Pi_{33} & 0 & 0 & 0 & \Pi_{37} & 0 & \Pi_{39} & 0 & 0 \\
* & * & * & \Pi_{44} & 0 & 0 & -N_{15}^{T} & 0 & -N_{16}^{T} & \Pi_{4,10} & 0 \\
* & * & * & * & \Pi_{55} & \Pi_{56} & -C^{T} N_{18}^{T} & -N_{17} G_{3} & -C^{T} N_{20}^{T} & 0 & 0 \\
* & * & * & * & * & \Pi_{66} & \Pi_{67} & \Pi_{68} & \widehat{\Pi}_{69} & 0 & 0 \\
* & * & * & * & * & * & \Pi_{77} & -N_{18} G_{3} & -N_{18} G_{4} & \Pi_{7,10} & 0 \\
* & * & * & * & * & * & * & \Pi_{88} & -G_{3}^{T} N_{20}^{T} & 0 & 0 \\
* & * & * & * & * & * & * & * & -\operatorname{sym}\left(N_{20} G_{4}\right) & \Pi_{9,10} & 0 \\
* & * & * & * & * & * & * & * & * & \Pi_{10,10} & 0 \\
* & * & * & * & * & * & * & * & * & * & \widehat{\Pi}_{11,11}
\end{array}\right]<0
$$

with

$$
\begin{aligned}
& \tilde{\Pi}_{11}=\operatorname{sym}\left(P^{T}\left(\bar{A}+\left(W_{1}^{T} H_{1}\left[\begin{array}{c}
0 \\
L_{1}
\end{array}\right]+W_{2}^{T} H_{2}\left[\begin{array}{c}
0 \\
L_{2}
\end{array}\right]\right)\left[\begin{array}{ll}
I & 0
\end{array}\right]\right)\right)+\Omega, \\
& \widehat{\Pi}_{1,11}=\left[h_{2} P^{T}\left(W_{1}^{T} H_{1}+I\right)^{T} \bar{H}_{1} \tau_{1} P^{T}\left(W_{2}^{T} H_{2}+I\right)^{T} \bar{H}_{2} h_{2}\left[\begin{array}{l}
0 \\
I
\end{array}\right]\left[\begin{array}{c}
0 \\
L_{1}
\end{array}\right]^{T} \tau_{1}\left[\begin{array}{l}
0 \\
I
\end{array}\right]^{0}\left[\begin{array}{c}
0 \\
L_{2}
\end{array}\right]^{T}\right] \text {, } \\
& \widehat{\Pi}_{11,11}=\operatorname{diag}\left\{-h_{2} \bar{H}_{1},-\tau_{1} \bar{H}_{2},-h_{2} \bar{H}_{1},-\tau_{1} \bar{H}_{2}\right\} \text {, }
\end{aligned}
$$

where $\bar{H}_{i}=H_{i}^{-1}(i=1,2)$.

Following [34,35], we choose $P_{3}=\delta P_{2}, \delta \in R$, where $\delta$ is a tuning scalar parameter (which may be restrictive). Let

$$
\zeta=\operatorname{diag}\{\underbrace{I, \ldots, I}_{16 \text { elements }}, \underbrace{P^{T}, \ldots, P^{T}}_{4 \text { elements }}\} .
$$

Premultiplying $\zeta$ and postmultiplying $\zeta^{T}$ to the matrix inequality (3.24) and considering $Y_{i}:=$ $P_{2}^{T} L_{i}, \widetilde{H}_{i}:=P^{T} \bar{H}_{i} P$, and $H_{i} W_{i}=\alpha_{i} I(i=1,2)$ to eliminate the nonlinearities in the matrix inequality, we obtain the LMI (3.3). Moreover, from (2.1) and the fact that $x(t)$ is square integrable on $[0, \infty)$, it follows that $D \eta_{t} \in L_{2}^{n}[0, \infty)$. Under (A1), the later implies that $\eta(t-$ $\tau(t)) \in L_{2}^{n}[0, \infty)$. Therefore, by [1, Theorem 1.6], we conclude that the neutral system (2.1) is asymptotically stable.

Remark 3.2. The results given in Theorem 3.1 are derived for system (2.1) with timevarying delays $h(t), \tau(t)$, and $r(t)$ satisfying (2.2a), (2.2b), and (2.2c), where the derivatives 
of the time-varying delays are available. However, in many situations, the information on the derivative of time-varying delays is unknown a prior. In such circumstances, the corresponding delay-rate-independent stability analysis results for time-delays only satisfying

$$
\begin{gathered}
0<h_{1} \leq h(t) \leq h_{2}<\infty, \\
0<\tau(t) \leq \tau_{1}<\infty, \\
0<r(t) \leq r_{1}<\infty,
\end{gathered}
$$

can be easily obtained by setting $Q_{1}=Q_{2}=Q_{4}=R_{3}=0$ in Theorem 3.1.

Remark 3.3. The reduced conservatism of Theorem 3.1 benefits from the construction of the new Lyapunov-Krasovskii functional in (3.6), utilizing Leibniz-Newton formula, using a free-weighting matrix technique, and no bounding technique is needed to estimate the inner product of the involved crossing terms (see, e.g., $[12,20]$ ). It can be easily seen that results of this paper are quite different from most existing results in the recent literature in the following perspectives. (a) Theoretically stability analysis of neutral systems with different time-varying neutral, discrete, and distributed delays is much more complicated, especially, for the case where the delays are time-varying and different. (b) In this paper, the derived sufficient conditions are convex, neutral-delay-dependent, discrete-delay-rangedependent, and distributed-delay-dependent, which make the treatment in the present paper more general with less conservative in compare to most existing results in the literature which are independent of the neutral or distributed delays; see for instance [21, 22, 38].

\section{Uncertainty Characterization}

In this section, we will discuss the uncertainty characterization for the linear neutral system (2.1) with different time-varying neutral, discrete, and distributed delays and nonlinear parameter perturbations.

\subsection{Polytopic Uncertainty}

The first class of uncertainty frequently encountered in practice is the polytopic uncertainty [2]. In this case, the matrices of the system (2.1) are not exactly known, except that they are within a compact set $\Omega$ denoting

$$
\Omega=\left[\begin{array}{llllll}
C & A & B & G_{1} & G_{2} & G_{3}
\end{array}\right] .
$$

We assume that

$$
\Omega=\sum_{j=1}^{N} s_{j} \Omega_{j}
$$


for some scalars $s_{j}$ satisfying

$$
0 \leq s_{j} \leq 1, \quad \sum_{j=1}^{N} s_{j}=1,
$$

where the $N$ vertices of the polytope are described by

$$
\Omega_{j}=\left[\begin{array}{llllll}
C^{(j)} & A^{(j)} & B^{(j)} & G_{1}^{(j)} & G_{2}^{(j)} & G_{3}^{(j)}
\end{array}\right] .
$$

In order to take into account the polytopic uncertainty in the system (2.1), we derive the following result from applying the same transformation that was used in deriving Theorem 3.1.

Theorem 4.1. Under (A1), for given scalars $\gamma, h_{1}, h_{2}, \tau_{1}, r_{1}>0, h_{3}, \tau_{2}, r_{2}$, if the uncertainty set $\Omega$ is polytopic with vertices $\Omega_{j}, j=1,2, \ldots, N$, then the system described by (2.1), (2.2a), $(2.2 \mathrm{~b}),(2.2 \mathrm{c})$, and (4.2)-(4.4) is asymptotically stable if there exist some scalars $\delta, \alpha_{1}, \alpha_{2}$, matrices $P_{2},\left\{N_{i}\right\}_{i=1}^{20}, Y_{1}, Y_{2}$, and positive-definite matrices $P_{1},\left\{Q_{i}\right\}_{i=1}^{4},\left\{R_{i}\right\}_{i=1}^{4}, \widetilde{H}_{1}, \widetilde{H}_{2}$ such that LMI (3.3) is satisfied for all

$$
\left[\begin{array}{llllll}
C & A & B & G_{1} & G_{2} & G_{3}
\end{array}\right]=\left[\begin{array}{llllll}
C^{(j)} & A^{(j)} & B^{(j)} & G_{1}^{(j)} & G_{2}^{(j)} & G_{3}^{(j)}
\end{array}\right], \quad j=1,2, \ldots, N .
$$

Proof. It follows directly from the proof of Theorem 3.1 and using properties of (4.2)-(4.4).

\subsection{Norm-Bounded Uncertainty}

There are also other uncertainties that cannot be reasonably modeled by a polytopic uncertainty set with a number of vertices. In such a case, it is assumed that the deviation of the system parameters of an uncertain system from their nominal values is norm bounded [2]. In our case, consider the time-varying structured uncertain neutral system

$$
\begin{aligned}
& \dot{x}(t)-(C+\Delta C(t)) \dot{x}(t-\tau(t))=(A+\Delta A(t)) x(t)+(B+\Delta B(t)) x(t-h(t)) \\
&+\left(G_{1}+\Delta G_{1}(t)\right) f_{1}(t, x(t)) \\
&+\left(G_{2}+\Delta G_{2}(t)\right) f_{2}(t, x(t-h(t))) \\
&+\left(G_{3}+\Delta G_{3}(t)\right) \int_{t-r(t)}^{t} f_{3}(\theta, x(\theta)) d \theta \\
&+\left(G_{4}+\Delta G_{4}(t)\right) f_{4}(t, \dot{x}(t-\tau(t))), \\
& x(t)=\phi(t), \quad t \in[-\kappa, 0],
\end{aligned}
$$


where the time-varying structured uncertainties are said to be admissible if the following form holds:

$$
\begin{gathered}
{\left[\begin{array}{ccccccc}
\Delta C(t) & \Delta A(t) & \Delta B(t) & \Delta G_{1}(t) & \Delta G_{2}(t) & \Delta G_{3}(t) & \Delta G_{4}(t)
\end{array}\right]} \\
=M_{1} \Delta(t)\left[\begin{array}{llllllll}
L_{c} & L_{a} & L_{b} & L_{g_{1}} & L_{g_{2}} & L_{g_{3}} & L_{g_{4}}
\end{array}\right]
\end{gathered}
$$

where $L_{c}, L_{a}, L_{b}, L_{g_{1}}, L_{g_{2}}, L_{g_{3}}, L_{g_{4}}$ are constant matrices with appropriate dimensions, $\Delta(t)$ is an unknown, real, and possibly time-varying matrix with Lebesgue measurable elements, and its Euclidean norm satisfies

$$
\|\Delta(t)\| \leq 1, \quad \forall t
$$

In this section, we modify (A1)-(A2) in order to enable the application of Lyapunov's method for the stability of the time-varying structured uncertain neutral system (4.6) as follows:

$\left(\mathrm{A}^{\prime} 1\right)$ let the difference operator $D: C\left([-\mathcal{\kappa}, 0], \mathfrak{R}^{n}\right) \rightarrow \mathfrak{R}^{n}$ given by $D x_{t}=x(t)-$ $(C+\Delta C(t)) x(t-\tau(t))$ be delay-independently stable with respect to all delays. A sufficient condition for $\left(\mathrm{A}^{\prime} 1\right)$ is that

$\left(\mathrm{A}^{\prime} 2\right)$ all the eigenvalues of the matrix $C+\Delta C(t)$ are inside the unit circle, that is, $\| C+$ $\Delta C(t) \|<1$.

Theorem 4.2. Under ( $\left.A^{\prime} 1\right)$, for given scalars $r, h_{1}, h_{2}, \tau_{1}, r_{1}>0, h_{3}, \tau_{2}, r_{2}$, the neutral system (4.6) with admissible uncertainties (4.7) and (4.8) is robustly asymptotically stable if there exist some scalars $\left\{\lambda_{i}\right\}_{i=1}^{4}>0, \delta, \alpha_{1}, \alpha_{2}$, matrices $P_{2},\left\{N_{i}\right\}_{i=1}^{20}, Y_{1}, Y_{2}$, and positive-definite matrices $P_{1},\left\{Q_{i}\right\}_{i=1}^{4},\left\{R_{i}\right\}_{i=1}^{4}, \widetilde{H}_{1}, \widetilde{H}_{2}$, such that the following LMI is feasible:

$$
\left[\begin{array}{ccc}
\Pi & \Psi_{1} & \Psi_{2} \\
* & -\Psi_{3} & 0 \\
* & * & -\Psi_{3}
\end{array}\right]<0
$$

where $\Psi_{1}=\left[\begin{array}{llll}\Gamma_{d_{1}} & \Gamma_{d_{2}} & \Gamma_{d_{3}} & \Gamma_{d_{4}}\end{array}\right], \Psi_{2}=\left[\begin{array}{llll}\lambda_{1} \Gamma_{e_{1}}^{T} & \lambda_{2} \Gamma_{e_{2}}^{T} & \lambda_{3} \Gamma_{e_{3}}^{T} & \lambda_{4} \Gamma_{e_{4}}^{T}\end{array}\right]$, and $\Psi_{3}=\operatorname{diag}\left\{\lambda_{1} I, \lambda_{2} I, \lambda_{3} I\right.$, $\left.\lambda_{4} I\right\}$ with

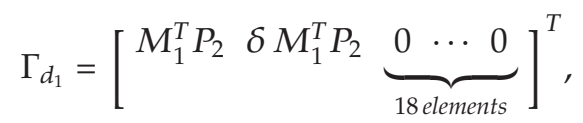

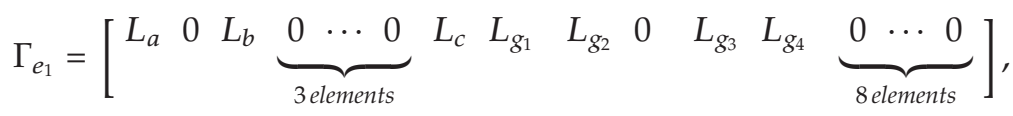

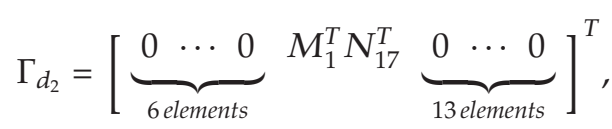

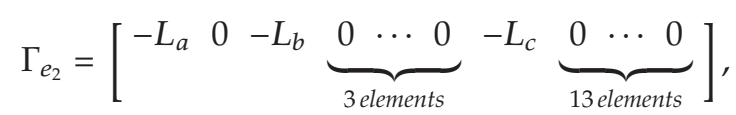




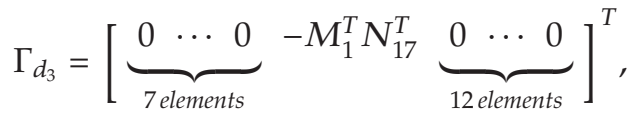

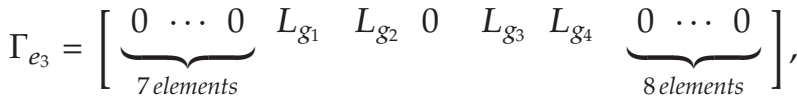

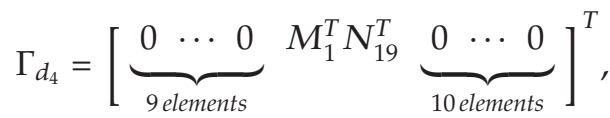

$$
\begin{aligned}
& \Gamma_{e_{4}}=\left[\begin{array}{llllllllll}
0 & 0 & -L_{b} & \underbrace{\begin{array}{lllllll}
0 & \cdots & 0
\end{array}}_{\text {4elements }}-L_{g_{1}} & -L_{g_{2}} & 0 & -L_{g_{3}} & -L_{g_{4}} & \underbrace{0 \cdots}_{\text {8elements }}
\end{array}\right] .
\end{aligned}
$$

Proof. If the matrices $C, A, B, G_{1}, G_{2}, G_{3}, G_{4}$ in (3.3) are replaced with $C+M_{1} \Delta(t) L_{c}, A+$ $M_{1} \Delta(t) L_{a}, B+M_{1} \Delta(t) L_{b}, G_{1}+M_{1} \Delta(t) L_{g_{1}}, G_{2}+M_{1} \Delta(t) L_{g_{2}}, G_{3}+M_{1} \Delta(t) L_{g_{3}}$, and $G_{4}+$ $M_{1} \Delta(t) L_{g_{4}}$, respectively, then (3.3) with the admissible uncertainties (4.7) is equivalent to the following condition:

$$
\Pi+\sum_{i=1}^{4} \operatorname{sym}\left(\Gamma_{d_{i}}^{T} \Delta(t) \Gamma_{e_{i}}\right)<0
$$

By Lemma 2.3, a necessary and sufficient condition for (4.11) is that there exist some $\left\{\lambda_{i}\right\}_{i=1}^{4}>$ 0 such that

$$
\Pi+\sum_{i=1}^{4}\left[\lambda_{i}^{-1} \Gamma_{d_{i}}^{T} \Gamma_{d_{i}}+\lambda_{i} \Gamma_{e_{i}}^{T} \Gamma_{e_{i}}\right]<0
$$

Applying Schur complements, we find that (4.12) is equivalent to (4.9).

Remark 4.3. It is noted that our approach is different from that in the reference [20] in several perspectives. (a) The system structure in [20] considers a system with different time-varying neutral and discrete delays and in compare to our case do not center on timevarying distributed delays, that is, the results in [20] cannot be directly applied to the systems with different time-varying neutral, discrete, and distributed delays. (b) Their system only considers the case that the range of the time-varying delay $h(t)$ is from 0 to an upper bound in compare to our case that the time-varying discrete delay $(h(t))$ lies in a range, in which the lower bound is not 0 . (c) The derived neutral-delay-range-independent conditions and using the inequality bounding technique [11, Lemma 1] employed for some cross terms encountered in their analysis conditions may produce conservative results in comparison with the present paper. 
Table 1: Comparative results for $h_{2}$.

\begin{tabular}{lcccc}
\hline & \multicolumn{2}{c}{$\eta_{1}=0, \eta_{2}=0.1$} & \multicolumn{2}{c}{$\eta_{1}=0.1, \eta_{2}=0.1$} \\
\hline & $h_{3}=0$ & $h_{3}=0.5$ & $h_{3}=0$ & $h_{3}=0.5$ \\
\hline Results of [10] & 0.6811 & 0.5467 & 0.6129 & 0.4950 \\
Results of [18] & 1.3279 & 0.6743 & 1.2503 & 0.5716 \\
Results of [21] & 2.742 & 1.142 & 1.875 & 1.009 \\
Results of [22] & 3.744 & 1.471 & 2.443 & 1.299 \\
\hline Results of this paper & 3.8205 & 1.6350 & 2.7105 & 1.3580 \\
\hline
\end{tabular}

Table 2: Comparative results for $h_{2}$.

\begin{tabular}{lcccc}
\hline & $\eta_{3}=0.0$ & $\eta_{3}=0.1$ & $\eta_{3}=0.2$ & $\eta_{3}=0.3$ \\
\hline Results of [9] & 0.7437 & 0.5131 & 0.3112 & 0.1398 \\
Results of [20] & 0.7749 & 0.5658 & 0.3859 & 0.2357 \\
\hline Results of this paper & 0.8429 & 0.6903 & 0.4504 & 0.3015 \\
\hline
\end{tabular}

\section{Numerical Results}

In this section, two examples are provided to illustrate the effectiveness of the results obtained in the previous sections.

Example 5.1. Consider the neutral system (2.1) with the following matrices adopted from [9]:

$$
A=\left[\begin{array}{cc}
-1.2 & 0.1 \\
-0.1 & -1
\end{array}\right], \quad B=\left[\begin{array}{cc}
-0.6 & 0.7 \\
-1 & -0.8
\end{array}\right], \quad G_{1}=G_{2}=I, \quad G_{3}=0
$$

and the nonlinear parameter perturbations $\left\|f_{1}(t, x(t))\right\| \leq \eta_{1}\|x(t)\|$ and $\left\|f_{2}(t, x(t-h(t)))\right\| \leq$ $\eta_{2}\|x(t-h(t))\|$, where $\eta_{1}, \eta_{2} \geq 0$.

Case 1. Assume that $C=0, h_{1}=0$, and $G_{4}=0$. Applying the criteria in $[10,18,21,22]$ and Theorem 3.1 in this paper, the maximum value of $h_{2}$ for stability of system under consideration is listed in Table 1. Furthermore, in the case of $h_{3}=0.5$ and $\eta_{1}=\eta_{2}=0.1$ by the criteria in [20], the nominal system is asymptotically stable for any $h(t)$ satisfying $h_{2}=1.0097$. It is easy to see that the stability criterion in this paper gives a much less conservative result than that in [10,20-22], and also the maximum value of $h_{2}$ decreases as $\eta_{i}$ increases. Moreover, unlike the results obtained in [10, 18, 21,22] our approach can also consider the case $h_{3} \geq 1$ and handle fast time-varying delays completely.

Case 2. Assume that $C=\operatorname{diag}\{0.1,0.1\}, G_{4}=I, h_{1}=0, h_{3}=\tau_{2}=0.5$, and $\left\|f_{4}(t, \dot{x}(t-\tau(t)))\right\| \leq$ $\eta_{3}\|\dot{x}(t-\tau(t))\|$. We also calculate the maximum delay bounds $h_{2}$ that guarantee the asymptotic stability of the system under consideration by Theorem 3.1 in this paper and developed methods in $[9,20]$ for different values of the parameter $\eta_{3}$, as listed in Table 2 . It can be seen from the table that $h_{2}$ decreases as $\eta_{3}$ increases, and the stability condition in Theorem 3.1 of this paper is less conservative than that in $[9,20]$. 
Table 3: Upper bounds of delays $h_{2}$ with respect to $r_{2}$.

\begin{tabular}{lccccc}
\hline$r_{2}$ & 0.1 & 0.3 & 0.5 & 0.7 & 0.9 \\
\hline$h_{2}$ & 1.6025 & 1.5875 & 1.5215 & 1.4050 & 1.1510 \\
\hline
\end{tabular}

Table 4: Upper bounds of delays $r_{2}$ with respect to $h_{2}$.

\begin{tabular}{lccccc}
\hline$h_{2}$ & 0.1 & 0.3 & 0.5 & 0.7 & 0.9 \\
\hline$r_{2}$ & 1.2560 & 1.2358 & 1.2125 & 1.1950 & 1.1045 \\
\hline
\end{tabular}

Example 5.2 (see [46]). Consider the system (4.6) with the following matrices:

$$
\begin{aligned}
& A=\left[\begin{array}{cc}
-2 & 0 \\
0 & -3
\end{array}\right], \quad G_{1}=\left[\begin{array}{cc}
0.5 & -0.1 \\
-0.2 & -0.3
\end{array}\right], \quad G_{2}=\left[\begin{array}{cc}
0.2 & -0.4 \\
0.1 & 0.2
\end{array}\right], \quad G_{3}=\left[\begin{array}{ll}
0.4 & 0.3 \\
0.1 & 0.2
\end{array}\right], \\
& L_{a}=\left[\begin{array}{cc}
0 & 0 \\
0.2 & 0.2
\end{array}\right], \quad L_{g_{1}}=\left[\begin{array}{cc}
0 & 0 \\
0.1 & 0.1
\end{array}\right], \quad L_{g_{2}}=\left[\begin{array}{cc}
0 & 0 \\
0.3 & 0.3
\end{array}\right], \quad L_{g_{3}}=\left[\begin{array}{cc}
0 & 0 \\
0.2 & 0.2
\end{array}\right] \text {, } \\
& B=C=G_{4}=L_{c}=L_{b}=L_{g_{4}}=0, \quad M_{1}=I, \quad U_{1}=U_{2}=U_{3}=I .
\end{aligned}
$$

Recently, Kwon and Park in [46] derived the stability bound of $h(t)=r(t)$ with $h_{3}=r_{2}=1$ as $0<h(t) \leq 1.97$ with the parameters above for the system (4.6). However, by applying Theorem 4.2 to the system under consideration, one can see that our criterion is feasible for $0<h(t) \leq 2.0105$. And for the condition $h_{3}=1$, for fixed $h_{2}$ or $r_{2}$, the upper bounds of delays $h_{2}$ and $r_{2}$ are shown in Tables 3 and 4, respectively. From Tables 3 and 4, it can be seen that Theorem 4.2 provides a condition for guaranteeing stability with respect to given delay bounds $h(t)$ and $r(t)$.

\section{Conclusion}

The problem of stability analysis has been presented in this paper for a class of neutral systems with different time-varying neutral, discrete, and distributed delays and nonlinear parameter perturbations using an appropriate Lyapunov-Krasovskii functional. By combining the descriptor model transformation, the Leibniz-Newton formula, some free-weighting matrices, and a suitable change of variables, new feasibility conditions, which are neutral-delay-dependent, discrete-delay-range-dependent, and distributed-delaydependent, have been developed to ensure that the considered system is asymptotically stable. The conditions were presented in terms of linear matrix inequalities (LMIs) and solved by existing convex optimization techniques. Two numerical examples were given to demonstrate the less conservatism of the proposed results over some existence results in the literature.

\section{Acknowledgments}

This work has been partially funded by the European Union (European Regional Development Fund) and the Ministry of Science and Innovation (Spain) through the coordinated 
research project DPI2008-06699-C02-01. H. R. Karimi is grateful to the grant of Juan de la Cierva program of the Ministry of Science and Innovation (Spain), and M. Zapateiro is grateful to the FI grant of the Department for Innovation, University and Enterprise of the Government of Catalonia (Spain). The authors would also like to thank the associate editor and anonymous reviewers for their constructive comments and suggestions to improve the quality of the paper.

\section{References}

[1] J. K. Hale and S. M. Verduyn Lunel, Introduction to Functional-Differential Equations, vol. 99 of Applied Mathematical Sciences, Springer, New York, NY, USA, 1993.

[2] K. Gu, V. L. Kharitonov, and J. Chen, Stability of Time-Delay Systems, Birkhäuser, Boston, Mass, USA, 2003.

[3] Y. Kuang, Delay Differential Equations with Applications in Population Dynamics, vol. 191 of Mathematics in Science and Engineering, Academic Press, Boston, Mass, USA, 1993.

[4] Y. Wang, L. Xie, and C. E. de Souza, "Robust control of a class of uncertain nonlinear systems," Systems E Control Letters, vol. 19, no. 2, pp. 139-149, 1992.

[5] H. R. Karimi, "A computational method for optimal control problem of time-varying state-delayed systems by Haar wavelets," International Journal of Computer Mathematics, vol. 83, no. 2, pp. 235-246, 2006.

[6] Y. He, Q.-G. Wang, L. Xie, and C. Lin, "Further improvement of free-weighting matrices technique for systems with time-varying delay," IEEE Transactions on Automatic Control, vol. 52, no. 2, pp. 293-299, 2007.

[7] Q.-L. Han, "A descriptor system approach to robust stability of uncertain neutral systems with discrete and distributed delays," Automatica, vol. 40, no. 10, pp. 1791-1796, 2004.

[8] E. Fridman, "New Lyapunov-Krasovskii functionals for stability of linear retarded and neutral type systems," Systems \& Control Letters, vol. 43, no. 4, pp. 309-319, 2001.

[9] Q.-L. Han and L. Yu, "Robust stability of linear neutral systems with nonlinear parameter perturbations," IEE Proceedings: Control Theory and Applications, vol. 151, no. 5, pp. 539-546, 2004.

[10] Q.-L. Han, "Robust stability for a class of linear systems with time-varying delay and nonlinear perturbations," Computers \& Mathematics with Applications, vol. 47, no. 8, pp. 1201-1209, 2004.

[11] P. Park, "A delay-dependent stability criterion for systems with uncertain time-invariant delays," IEEE Transactions on Automatic Control, vol. 44, no. 4, pp. 876-877, 1999.

[12] Y. S. Moon, P. Park, W. H. Kwon, and Y. S. Lee, “Delay-dependent robust stabilization of uncertain state-delayed systems," International Journal of Control, vol. 74, no. 14, pp. 1447-1455, 2001.

[13] Ju. H. Park and O. Kwon, "On new stability criterion for delay-differential systems of neutral type," Applied Mathematics and Computation, vol. 162, no. 2, pp. 627-637, 2005.

[14] Y. He, Q.-G. Wang, C. Lin, and M. Wu, "Delay-range-dependent stability for systems with timevarying delay," Automatica, vol. 43, no. 2, pp. 371-376, 2007.

[15] Q.-L. Han, X. Yu, and K. Gu, “On computing the maximum time-delay bound for stability of linear neutral systems," IEEE Transactions on Automatic Control, vol. 49, no. 12, pp. 2281-2285, 2004.

[16] X. Jiang and Q.-L. Han, "Delay-dependent robust stability for uncertain linear systems with interval time-varying delay," Automatica, vol. 42, no. 6, pp. 1059-1065, 2006.

[17] J. Lam, H. Gao, and C. Wang, " $H_{\infty}$ model reduction of linear systems with distributed delay," IEE Proceedings: Control Theory and Applications, vol. 152, no. 6, pp. 662-674, 2005.

[18] Y.-Y. Cao and J. Lam, "Computation of robust stability bounds for time-delay systems with nonlinear time-varying perturbations," International Journal of Systems Science, vol. 31, no. 3, pp. 359-365, 2000.

[19] Y. Chen, A. Xue, R. Lu, and S. Zhou, "On robustly exponential stability of uncertain neutral systems with time-varying delays and nonlinear perturbations," Nonlinear Analysis: Theory, Methods $\mathcal{E}$ Applications, vol. 68, no. 8, pp. 2464-2470, 2008.

[20] M. N. A. Parlakçı, “Delay-dependent robust stability criteria for uncertain neutral systems with mixed time-varying discrete and neutral delays," Asian Journal of Control, vol. 9, no. 4, pp. 411-421, 2007.

[21] J. Zhang, P. Shi, and J. Qiu, "Robust stability criteria for uncertain neutral system with time delay and nonlinear uncertainties," Chaos, Solitons \& Fractals, vol. 38, no. 1, pp. 160-167, 2008. 
[22] C.-C. Shen and S.-M. Zhong, "New delay-dependent robust stabilitycriterion for uncertain neutral systems with time-varying delay and nonlinear uncertainties," Chaos, Solitons E Fractals, 2007.

[23] Y. He, G.-P. Liu, D. Rees, and M. Wu, "Improved delay-dependent stability criteria for systems with nonlinear perturbations," European Journal of Control, vol. 13, no. 4, pp. 356-365, 2007.

[24] B. Yang, J. Wang, X. Pan, and C. Zhong, "Delay-dependent criteria for robust stability of linear neutral systems with time-varying delay and nonlinear perturbations," International Journal of Systems Science, vol. 38, no. 6, pp. 511-518, 2007.

[25] Y. Chen and W. Su, "New robust stability of cellular neural networks with time-varying discrete and distributed delays," International Journal of Innovative Computing, Information and Control, vol. 3, no. 6B, pp. 1549-1556, 2007.

[26] W.-H. Chen and W. X. Zheng, "Delay-dependent robust stabilization for uncertain neutral systems with distributed delays," Automatica, vol. 43, no. 1, pp. 95-104, 2007.

[27] Z. Zuo and Y. Wang, "New stability criterion for a class of linear systems with time-varying delay and nonlinear perturbations," IEE Proceedings: Control Theory and Applications, vol. 153, no. 5, pp. 623-626, 2006.

[28] J. D. Chen, C. H. Lien, K. K. Fan, and J. H. Chou, “Criteria for asymptotic stability of a class of neutral systems viaa LMI approach," IEE Proceedings: Control Theory and Applications, vol. 148, no. 6, pp. 442 447, 2001.

[29] J. D. Chen, "LMI-based robust $H_{\infty}$ control of uncertain neutral systems with state and input delays," Journal of Optimization Theory and Applications, vol. 126, no. 3, pp. 553-570, 2005.

[30] H. R. Karimi, P. J. Maralani, B. Lohmann, and B. Moshiri, " $H_{\infty}$ control of parameter-dependent statedelayed systems using polynomial parameter-dependent quadratic functions," International Journal of Control, vol. 78, no. 4, pp. 254-263, 2005.

[31] M. Basin, J. Perez, and D. Calderon-Alvarez, "Optimal filtering for linear systems over polynomial observations," International Journal of Innovative Computing, Information and Control, vol. 4, no. 2, pp. 313-320, 2008.

[32] M. Basin, E. Sanchez, and R. Martinez-Zuniga, "Optimal linear filtering for systems with multiple state and observation delays," International Journal of Innovative Computing, Information and Control, vol. 3, no. 5, pp. 1309-1320, 2007.

[33] S. Xu, J. Lam, and C. Yang, " $H_{\infty}$ and positive-real control for linear neutral delay systems," IEEE Transactions on Automatic Control, vol. 46, no. 8, pp. 1321-1326, 2001.

[34] H. R. Karimi, M. Zapateiro, and N. Luo, "Robust mixed $H_{2} / H_{\infty}$ delayed state feedback control of uncertain neutral systems with time-varying delays," Asian Journal of Control, vol. 10, no. 5, pp. 569$580,2008$.

[35] H. R. Karimi, “Observer-based mixed $\mathrm{H}_{2} / \mathrm{H}_{\infty}$ control design for linear systems with time-varying delays: an LMI approach," International Journal of Control, Automation and Systems, vol. 6, no. 1, pp. 1-14, 2008.

[36] H. R. Karimi and H. Gao, "LMI-based delay-dependent mixed $H_{2} / H_{\infty}$ control of second-order neutral systems with time-varying state and input delays," ISA Transactions, vol. 47, no. 3, pp. 311324, 2008.

[37] B. Chen, J. Lam, and S. Xu, "Memory state feedback guaranteed cost control for neutral systems," International Journal of Innovative Computing, Information and Control, vol. 2, no. 2, pp. 293-303, 2006.

[38] Ju. H. Park, "Dynamic output guaranteed cost controller for neutral systems with input delay," Chaos, Solitons E Fractals, vol. 23, no. 5, pp. 1819-1828, 2005.

[39] M. S. Mahmoud, Y. Shi, and H. N. Nounou, "Resilient observer-based control of uncertain time-delay systems," International Journal of Innovative Computing, Information and Control, vol. 3, no. 2, pp. 407$418,2007$.

[40] D. D. Šiljak and D. M. Stipanović, "Robust stabilization of nonlinear systems: the LMI approach," Mathematical Problems in Engineering, vol. 6, no. 5, pp. 461-493, 2000.

[41] Z. Zuo, J. Wang, and L. Huang, "Robust stabilization for non-linear discrete-time systems," International Journal of Control, vol. 77, no. 4, pp. 384-388, 2004.

[42] Y. He, M. Wu, J.-H. She, and G.-P. Liu, "Delay-dependent robust stability criteria for uncertain neutral systems with mixed delays," Systems \& Control Letters, vol. 51, no. 1, pp. 57-65, 2004.

[43] X. He, Z. Wang, and D. Zhou, "Robust $H_{\infty}$ filtering for networked systems with multiple state delays," International Journal of Control, vol. 80, no. 8, pp. 1217-1232, 2007. 
[44] H. Gao, T. Chen, and J. Lam, "A new delay system approach to network-based control," Automatica, vol. 44, no. 1, pp. 39-52, 2008.

[45] P. P. Khargonekar, I. R. Petersen, and K. Zhou, "Robust stabilization of uncertain linear systems: quadratic stabilizability and $H_{\infty}$ control theory," IEEE Transactions on Automatic Control, vol. 35, no. 3, pp. 356-361, 1990.

[46] O. M. Kwon and Ju. H. Park, "Exponential stability for uncertain cellular neural networks with discrete and distributed time-varying delays," Applied Mathematics and Computation, vol. 203, no. 2, pp. 813-823, 2008. 


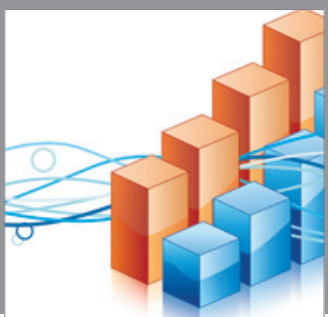

Advances in

Operations Research

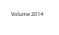

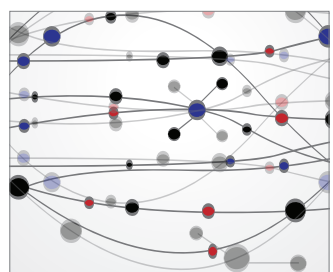

\section{The Scientific} World Journal
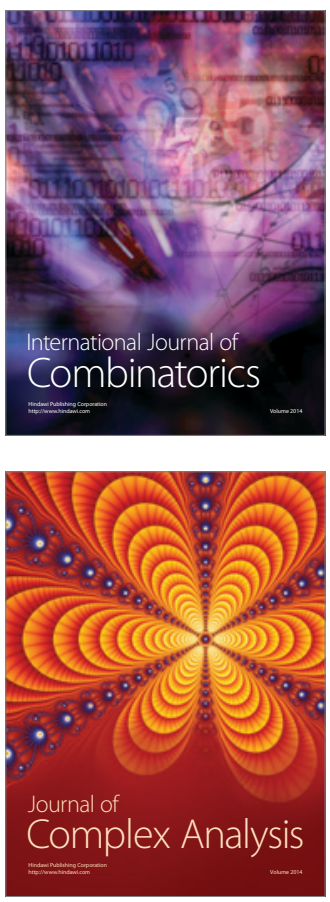

International Journal of

Mathematics and

Mathematical

Sciences
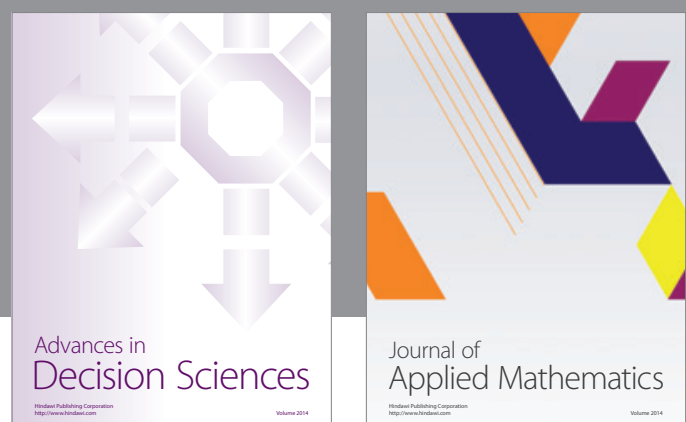

Journal of

Applied Mathematics
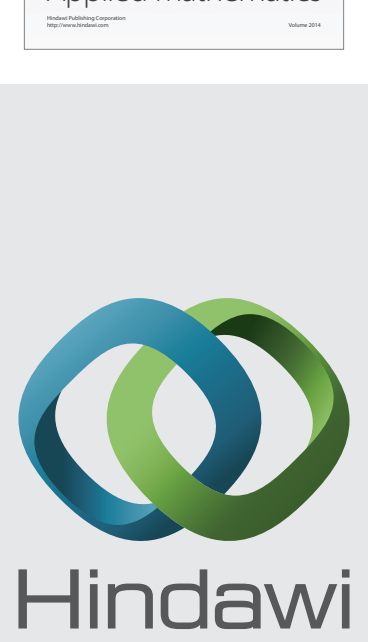

Submit your manuscripts at http://www.hindawi.com
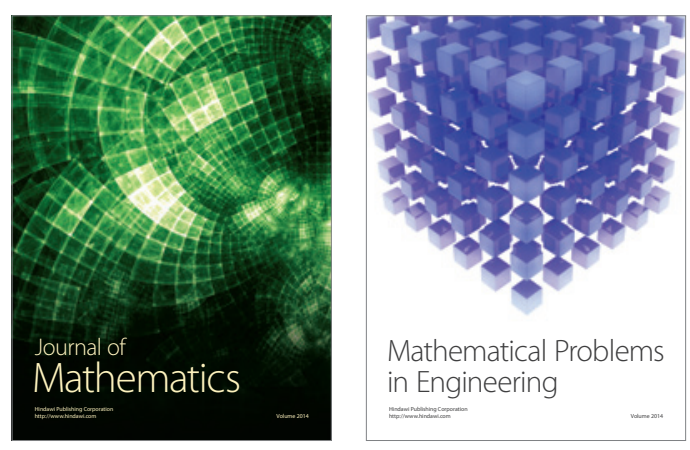

Mathematical Problems in Engineering
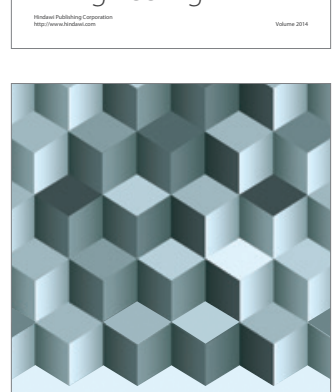

Journal of

Function Spaces
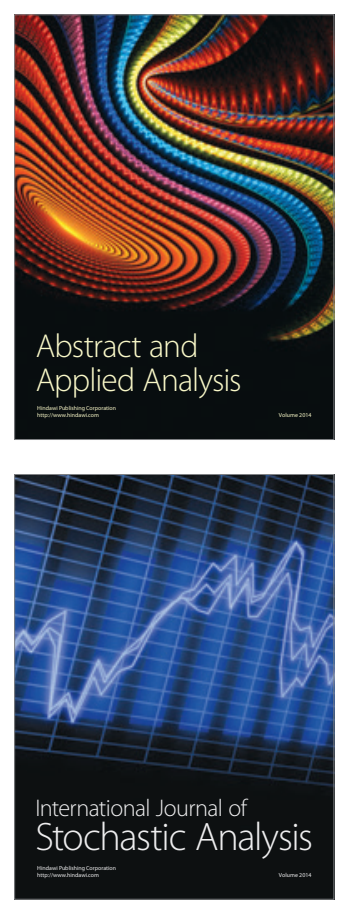

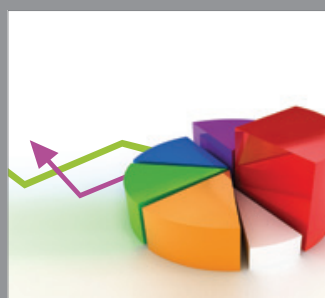

ournal of

Probability and Statistics

Promensencen
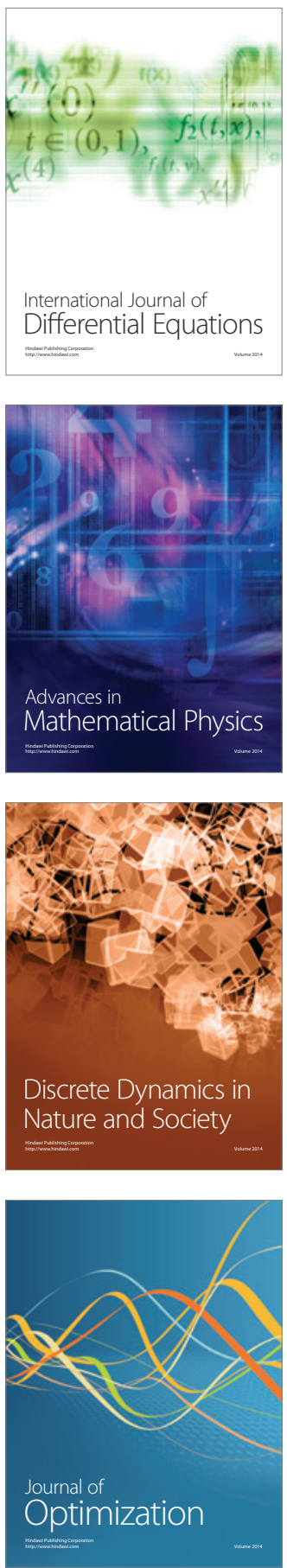